\title{
Semiclassical 3D Neumann Laplacian with variable magnetic field: a toy model
}

\author{
Nicolas Raymond
}

March 20, 2012

\begin{abstract}
In this paper we investigate the semiclassical behavior of the lowest eigenvalues of a model Schrödinger operator with variable magnetic field. This work aims at proving an accurate asymptotic expansion for these eigenvalues, the corresponding upper bound being already proved in the general case. The present work also aims at establishing localization estimates for the attached eigenfunctions.
\end{abstract}

\section{Motivation and main results}

\subsection{Motivation}

In this paper we are interested in a toy model of Schrödinger operator with variable magnetic field (with Dirichlet and Neumann conditions on the boundary) in the bounded region

$$
\Omega_{0}=\left\{(x, y, z) \in \mathbb{R}^{3}:|x| \leq x_{0},|y| \leq y_{0} \text { and } 0<z \leq z_{0}\right\},
$$

where $x_{0}, y_{0}, z_{0}>0$. We will need the following notation for the part of the boundary carrying the Dirichlet condition:

$$
\partial_{\text {Dir }} \Omega_{0}=\left\{(x, y, z) \in \Omega_{0}:|x|=x_{0} \text { or }|y|=y_{0} \text { or } z=z_{0}\right\} .
$$

Definition of the operator For $h>0, \alpha \geq 0$ and $\theta \in\left(0, \frac{\pi}{2}\right)$, we consider the self-adjoint operator:

$$
\mathcal{L}(h, \alpha, \theta)=h^{2} D_{y}^{2}+h^{2} D_{z}^{2}+\left(h D_{x}+z \cos \theta-y \sin \theta+\alpha z\left(x^{2}+y^{2}\right)\right)^{2},
$$

with domain:

$$
\begin{aligned}
\operatorname{Dom}(\mathcal{L}(h, \alpha, \theta))=\left\{\psi \in L^{2}\left(\Omega_{0}\right):\right. & \mathcal{L}(h, \alpha, \theta) \psi \in L^{2}\left(\Omega_{0}\right) \\
& \left.\psi=0 \text { on } \partial_{\operatorname{Dir}} \Omega_{0} \text { and } \partial_{z} \psi=0 \text { on } z=0\right\} .
\end{aligned}
$$


We will denote by $\left(\lambda(h), u_{h}\right)$ an eigenpair (we omit the dependence on $\alpha$ and $\theta$ ). The vector potential is expressed as:

$$
\mathbf{A}(x, y, z)=\left(V_{\theta}(y, z)+\alpha z\left(x^{2}+y^{2}\right), 0,0\right)=(A, 0,0)
$$

where

$$
V_{\theta}(y, z)=z \cos \theta-y \sin \theta .
$$

The associated magnetic field is given by:

$$
\nabla \times \mathbf{A}=\boldsymbol{\beta}=\left(0, \cos \theta+\alpha\left(x^{2}+y^{2}\right), \sin \theta-2 \alpha y z\right) .
$$

Constant magnetic field case $(\alpha=0) \quad$ Let us examine the important case when $\alpha=0$ :

$$
\mathcal{L}(h, 0, \theta)=h^{2} D_{y}^{2}+h^{2} D_{z}^{2}+\left(h D_{x}+V_{\theta}(y, z)\right)^{2}
$$

viewed as an operator on $L^{2}\left(\mathbb{R}_{+}^{3}\right)$. We perform the rescaling:

$$
x=h^{1 / 2} r, \quad y=h^{1 / 2} s, \quad z=h^{1 / 2} t
$$

and the operator becomes (after division by $h$ ):

$$
\mathcal{H}(\theta)=D_{s}^{2}+D_{t}^{2}+\left(D_{r}+V_{\theta}(s, t)\right)^{2} .
$$

Making a Fourier transform in the variable $r$ denoted by $\mathcal{F}$, we get:

$$
\mathcal{F H}(\theta) \mathcal{F}^{-1}=D_{s}^{2}+D_{t}^{2}+\left(\tau+V_{\theta}(s, t)\right)^{2} .
$$

Then, we use a change of coordinates:

$$
U_{\theta}(\tau, s, t)=(\hat{\tau}, \hat{s}, \hat{t})=\left(\tau, s-\frac{\tau}{\sin \theta}, t\right)
$$

and we obtain:

$$
\mathcal{H}^{\mathrm{Neu}}(\theta)=U_{\theta} \mathcal{F} \mathcal{H}(\theta) \mathcal{F}^{-1} U_{\theta}^{-1}=D_{\hat{s}}^{2}+D_{\hat{t}}^{2}+V_{\theta}(\hat{s}, \hat{t})^{2}
$$

Notation 1.1 We denote by $\mathcal{Q}_{\theta}$ the quadratic form associated with $\mathcal{H}^{\mathrm{Neu}}(\theta)$.

The operator $H^{\mathrm{Neu}}(\theta) \quad$ We can consider $\mathcal{H}^{\mathrm{Neu}}(\theta)$ as an operator acting on $L^{2}\left(\mathbb{R}_{+}^{2}\right)$; we denote by $H^{\mathrm{Neu}}(\theta)$ this realization. The bottom of its spectrum is denoted by $\sigma(\theta)$. In [10] (see the references therein, in particular [16, 19]), it is proved that $\sigma$ is analytic and strictly increasing on $] 0, \frac{\pi}{2}\left[\right.$, that $\sigma(0) \in(0,1), \sigma\left(\frac{\pi}{2}\right)=1$ and $\sigma_{\text {ess }}\left(H^{\mathrm{Neu}}(\theta)\right)=[1,+\infty[$. Therefore, $\sigma(\theta)$ is a simple eigenvalue. 
Notation 1.2 We denote by $u_{\theta}$ the positive and $L^{2}$-normalized eigenfunction (which is in the Schwartz class, see [24]) associated with $\sigma(\theta)$.

Let us also recall that the lower bound of the essential spectrum is related, through the Persson's theorem (see [22]), to the following estimate:

$$
q_{\theta}^{\mathrm{Neu}}\left(\chi_{R} u\right) \geq(1-\varepsilon(R))\left\|\chi_{R} u\right\|, \quad \forall u \in \operatorname{Dom}\left(q_{\theta}^{\mathrm{Neu}}\right),
$$

where $q_{\theta}^{\mathrm{Neu}}$ is the quadratic form associated with $H^{\mathrm{Neu}}(\theta)$, where $\chi_{R}$ is a cutoff function away from the ball $B(0, R)$ and $\varepsilon(R)$ is tending to zero when $R$ tends to infinity. Moreover, if we consider the Dirichlet realization $H^{\operatorname{Dir}}(\theta)$, we have:

$$
q_{\theta}^{\operatorname{Dir}}(u) \geq\|u\|^{2}, \quad \forall u \in \operatorname{Dom}\left(q_{\theta}^{\text {Dir }}\right) .
$$

Let us finally mention that an accurate analysis of the eigenpairs of $H^{\mathrm{Neu}}(\theta)$ is done in [5] in the regime $\theta \rightarrow 0$.

Motivations Let us explain why we are led to consider this model.

The reason comes from the theory of superconductivity. As much in $2 \mathrm{D}$ as in $3 \mathrm{D}$, the magnetic Laplacian appears in this theory when studying the third critical field $H_{C_{3}}$ (which distinguish between normal and superconducting states) after the linearization of the Ginzburg-Landau functional (see for instance [18, 19] and also the book of Fournais and Helffer [10]). It turns out that $H_{C_{3}}$ can be related to the lowest eigenvalue of the magnetic Laplacian in the regime $h \rightarrow 0$. Many properties of the eigenpairs are investigated when the magnetic field is constant (see [3, 4, 7, 15,9] for the 2D and [17] for the 3D), but less are known when the magnetic field is variable (see [18, 23] for the $2 \mathrm{D}$ and $[19,24]$ for the 3D). When the magnetic vanishes, we can refer to [20, 14, 21, 13, 8].

Let us introduce the fundamental invariant in the case of variable magnetic field and our generic assumptions. We let:

$$
\hat{\boldsymbol{\beta}}(x, y)=\sigma(\theta(x, y))\|\boldsymbol{\beta}(x, y, 0)\|,
$$

where $\theta(x, y)$ is the angle of $\boldsymbol{\beta}(x, y, 0)$ with the boundary $z=0$ :

$$
\|\boldsymbol{\beta}(x, y, 0)\| \sin \theta(x, y)=\boldsymbol{\beta}(x, y, 0) \cdot \nu(x, y),
$$

where $\nu(x, y)$ is the inward normal at $(x, y, 0)$. It is proved in [19] that the semiclassical asymptotics of the lowest eigenvalue is:

$$
\lambda_{1}(h)=\min \left(\inf _{z=0} \hat{\boldsymbol{\beta}}, \inf _{\Omega_{0}}\|\boldsymbol{\beta}\|\right) h+o(h) .
$$

In this paper (like in [24]), we are interested in the case when the following generic assumptions are satisfied:

$$
\inf _{z=0} \hat{\boldsymbol{\beta}}<\inf _{\Omega_{0}}\|\boldsymbol{\beta}\|
$$


$\hat{\boldsymbol{\beta}}$ admits a unique and non degenerate minimum.

Under these assumptions, a three terms upper bound is proved for $\lambda_{1}(h)$ in [24] and the corresponding lower bound, for a general domain, is still an open problem. The aim of this paper is to establish such a lower bound for an example. For $\alpha>0$, the toy operator (1.1) is the simplest example of a generic Schrödinger operator with variable magnetic field satisfying Assumptions (1.8) and (1.9). Let us explain in which sense. In the general case studied in [24] and after using Taylor formulas near $(0,0,0)$ at the order 3 , we are reduced, in the case of a flat metrics, to an operator in the form:

$$
h^{2} D_{z}^{2}+\left(h D_{y}+A_{y}^{(3)}\right)^{2}+\left(h D_{x}+A_{x}^{(3)}\right)^{2} .
$$

It turns out that the terms $A_{y}^{(3)}$ and the terms of order 2 of $A_{x}^{(3)}$ are not crucial in order to satisfy the generic assumptions: The most important come from the terms of order 3 in $A_{x}^{(3)}$. Then, we cancel a few more terms to get a diagonal matrix for the Hessian of $\hat{\beta}$. Thus, this model is the one with the less terms and so that the Hessian matrix of $\hat{\beta}$ is diagonal. Let us now verify the assumptions. Using the computations of [24], we have the Taylor expansion:

$$
\hat{\boldsymbol{\beta}}(x, y)=\sigma(\theta)+\alpha C(\theta)\left(x^{2}+y^{2}\right)+O\left(|x|^{3}+|y|^{3}\right) .
$$

with:

$$
C(\theta)=\cos \theta \sigma(\theta)-\sin \theta \sigma^{\prime}(\theta)
$$

Moreover, it is proved in [5, Proposition 5.1] that $C(\theta)>0$, for $\theta \in\left(0, \frac{\pi}{2}\right)$. Thus, Assumption (1.9) is verified if $x_{0}, y_{0}$ and $z_{0}$ are fixed small enough. Using $\sigma(\theta)<1$ when $\theta \in\left(0, \frac{\pi}{2}\right)$ and $\|\boldsymbol{\beta}(0,0,0)\|=1$, we get Assumption (1.8).

Finally, we can briefly compare to what is done in [17] in the case of the constant magnetic field. In their paper, the authors establish many localizations properties through Agmon estimates and reduce the general operator to simplest models. By doing this, they refine the localization properties and are led to a normal form involving an harmonic oscillator (see [17, Subsection 13.8]). This will be the main philosophy of this paper, even if we already start from a simplest operator.

Remark on the function $\hat{\boldsymbol{\beta}}$ Using the explicit expression of the magnetic field, we have:

$$
\hat{\boldsymbol{\beta}}(x, y)=\hat{\boldsymbol{\beta}}_{\text {rad }}(R), \quad R=\alpha\left(x^{2}+y^{2}\right)
$$

and an easy computation gives:

$$
\hat{\boldsymbol{\beta}}_{\text {rad }}(R)=\left\|\boldsymbol{\beta}_{\text {rad }}(R)\right\| \sigma\left(\arctan \left(\frac{\sin \theta}{\cos \theta+R}\right)\right),
$$

with

$$
\left\|\boldsymbol{\beta}_{\mathrm{rad}}(R)\right\|=\sqrt{(\cos \theta+R)^{2}+\sin ^{2} \theta} .
$$


The computations of [5, Proposition 5.1] imply that $\hat{\boldsymbol{\beta}}_{\text {rad }}$ is strictly increasing and

$$
\partial_{R} \hat{\boldsymbol{\beta}}_{\text {rad }}(R=0)=C(\theta)>0 .
$$

Consequently, $\hat{\boldsymbol{\beta}}$ admits a unique and non degenerate minimum on $\mathbb{R}_{+}^{3}$ and tends to infinity far from 0 . This is easy to see that:

$$
\inf _{\mathbb{R}_{+}^{3}}\|\boldsymbol{\beta}\|=\cos \theta .
$$

We deduce that, as long as $\sigma(\theta)<\cos \theta$, the generic assumptions of [24] are satisfied with $\Omega_{0}=\mathbb{R}_{+}^{3}$.

\subsection{Recall and improvement of existing results and statement of the main theorem}

Let us recall and improve the main result of [24] in our simplified case. We perform the scaling (1.4) and, after division by $h, \mathcal{L}(h, \alpha, \theta)$ becomes:

$$
\mathcal{L}^{\text {resc }}(h, \alpha, \theta)=D_{s}^{2}+D_{t}^{2}+\left(D_{r}+t \cos \theta-s \sin \theta+h \alpha t\left(r^{2}+s^{2}\right)\right) .
$$

Using the Fourier transform $\mathcal{F}$ (see (1.5)) and the translation $U_{\theta}$ (see (1.6)), we have:

$U_{\theta} \mathcal{F} \mathcal{L}^{\text {resc }}(h, \alpha, \theta) \mathcal{F}^{-1} U_{\theta}^{-1}=D_{\hat{s}}^{2}+D_{\hat{t}}^{2}+\left(V_{\theta}(\hat{s}, \hat{t})+h \alpha \hat{t}\left(\left(D_{\hat{\tau}}-\frac{D_{\hat{s}}}{\sin \theta}\right)^{2}+\left(\hat{s}+\frac{\hat{\tau}}{\sin \theta}\right)^{2}\right)^{2}\right.$.

This operator will be shortly denoted by $\mathcal{L}^{\text {Normal }}(h)$ and the corresponding quadratic form $q_{h}^{\text {Normal }}$. We write:

$$
\mathcal{L}^{\text {Normal }}(h)=H^{\text {Neu }}(\theta)+h H_{1}+h^{2} H_{2},
$$

where:

$$
\begin{gathered}
H_{1}=\alpha \hat{t}\left\{\left(D_{\hat{\tau}}-\frac{D_{\hat{s}}}{\sin \theta}\right)^{2} V_{\theta}+V_{\theta}\left(D_{\hat{\tau}}-\frac{D_{\hat{s}}}{\sin \theta}\right)^{2}+2 V_{\theta}\left(\hat{s}+\frac{\hat{\tau}}{\sin \theta}\right)^{2}\right\}, \\
H_{2}=\alpha^{2} \hat{t}^{2}\left\{\left(D_{\hat{\tau}}-\frac{D_{\hat{s}}}{\sin \theta}\right)^{2}+\left(\hat{s}+\frac{\tau}{\sin \theta}\right)^{2}\right\}^{2} \geq 0 .
\end{gathered}
$$

We look for quasi-eigenpairs in the form:

$$
\mu \sim \sum_{j \geq 0} \mu_{j} h^{j}
$$

and

$$
\psi \sim \sum_{j \geq 0} \psi_{j} h^{j}
$$

We solve the following problem in the sense of formal series:

$$
\mathcal{L}^{\text {Normal }}(h) \psi \sim \mu \psi \text {. }
$$


Term in $h^{0} \quad$ We solve:

$$
H(\theta) \psi_{0}=\mu_{0} \psi_{0} .
$$

We take: $\mu_{0}=\sigma(\theta)$ and:

$$
\psi_{0}(\hat{\tau}, \hat{s}, \hat{t})=u_{\theta}(\hat{s}, \hat{t}) f_{0}(\hat{\tau})
$$

$f_{0}$ being to be determined.

Term in $h$ Then, we must solve:

$$
(H(\theta)-\sigma(\theta)) \psi_{1}=\left(\mu_{1}-H_{1}\right) \psi_{0} .
$$

We apply the Fredholm alternative and we write:

$$
\left\langle\left(\mu_{1}-H_{1}\right) \psi_{0}, u_{\theta}\right\rangle_{\hat{s}, \hat{t}}=0 .
$$

Let us introduce a fundamental operator involved in the asymptotics. We let:

$$
\mathfrak{S}_{\theta}\left(D_{\hat{\tau}}, \hat{\tau}\right)=\left(2 \int \hat{t} V_{\theta} u_{\theta}^{2} d \hat{s} d \hat{t}\right) H_{\text {harm }}+\left(\frac{2}{\sin \theta} \int \hat{t} V_{\theta} u_{\theta}^{2} d \hat{s} d \hat{t}\right) \hat{\tau}+d(\theta),
$$

where

$$
H_{\text {harm }}=D_{\hat{\tau}}^{2}+\frac{\hat{\tau}^{2}}{\sin ^{2} \theta}
$$

and

$$
d(\theta)=\sin ^{-2} \theta\left\langle\hat{t}\left(D_{\hat{s}}^{2} V_{\theta}+V_{\theta} D_{\hat{s}}^{2}\right) u_{\theta}, u_{\theta}\right\rangle+2 \int \hat{t} \hat{s}^{2} V_{\theta} u_{\theta}^{2} d \hat{s} d \hat{t} .
$$

We recall the important fact that (see [24, Formula (2.31)]):

$$
2 \int t V_{\theta} u_{\theta}^{2} d s d t=C(\theta)>0
$$

so that $\mathfrak{S}_{\theta}\left(D_{\hat{\tau}}, \hat{\tau}\right)$ can be viewed as the harmonic oscillator up to dilation and translation. We denote $\nu_{n}\left(\mathfrak{S}_{\theta}\left(D_{\hat{\tau}}, \hat{\tau}\right)\right)$ the $n$-th eigenvalue of $\mathfrak{S}_{\theta}\left(D_{\hat{\tau}}, \hat{\tau}\right)$. The compatibility equation rewrites:

$$
\mathfrak{S}_{\theta}\left(D_{\hat{\tau}}, \hat{\tau}\right) f_{0}=\mu_{1} f_{0}
$$

and we take $\mu_{1}=\nu_{n}\left(\mathfrak{S}_{\theta}\left(D_{\hat{\tau}}, \hat{\tau}\right)\right)$ and for $f_{0}$ the corresponding $L^{2}$-normalized eigenfunction. Then, we can write the solution $\psi_{1}$ in the form:

$$
\psi_{1}=\psi_{1}^{\perp}+f_{1}(\hat{\tau}) u_{\theta}(\hat{s}, \hat{t})
$$

where $\psi_{1}^{\perp}$ is the unique solution orthogonal to $u_{\theta}$. We notice that it is in the Schwartz class. 
Further terms ("Grushin procedure") We perform a recursion to get the next terms (this is related to the so-called Grushin procedure, see [11]). We assume that $\psi_{k}$ is determined in the Schwartz class for $k=0, \cdots, n-1$ and that $\psi_{n}$ is written in the form:

$$
\psi_{n}=\psi_{n}^{\perp}+f_{n}(\hat{\tau}) u_{\theta}(\hat{s}, \hat{t})
$$

where $\psi_{n}^{\perp}$ is determined in the Schwartz class and where $f_{n}$ is to be determined. We also assume that $\mu_{k}$ is determined for $k=0, \cdots, n$. The equation for $h^{n+1}$ is in the form:

$$
(H(\theta)-\sigma(\theta)) \psi_{n+1}=\left(\mu_{1}-H_{1}\right)\left(f_{n} u_{\theta}\right)+\mu_{n+1} \psi_{0}+R_{n}(\hat{\tau}, \hat{s}, \hat{t})
$$

where $R_{n}(\hat{\tau}, \hat{s}, \hat{t})$ is determined and in the Schwartz class. The Fredholm alternative provides:

$$
\left(\mathfrak{S}_{\theta}\left(D_{\hat{\tau}}, \hat{\tau}\right)-\mu_{1}\right) f_{n}=\mu_{n+1} f_{0}(\hat{\tau})+r_{n}(\hat{\tau}), \text { with } r_{n}=\left\langle R_{n}, u_{\theta}\right\rangle_{\hat{s}, \hat{t}}
$$

Applying again the Fredholm alternative, we deduce that $\mu_{n+1}=-\left\langle r_{n}, f_{0}\right\rangle_{\hat{\tau}}$ permits to determine a unique solution $f_{n}$ in the Schwartz class which is orthogonal to $f_{0}$. As a consequence, we can write:

$$
\psi_{n+1}=\psi_{n+1}^{\perp}+f_{n+1}(\hat{\tau}) u_{\theta}(\hat{s}, \hat{t}) .
$$

Thus, we have determined $\left(\mu_{j}\right)_{j \geq 0}$ and $\left(\psi_{j}\right)_{j \geq 0}$. To emphasize the dependence on $n$, we shall write $\left(\mu_{j, n}\right)_{j \geq 0}$ and $\left(\psi_{j, n}\right)_{j \geq 0}$. Thanks to the spectral theorem, we deduce:

Theorem 1.3 For all $\alpha>0, \theta \in\left(0, \frac{\pi}{2}\right)$, there exists a sequence $\left(\mu_{j, n}\right)_{j \geq 0}$ and there exist positive constants $C, h_{0}$ such that for $h \in\left(0, h_{0}\right)$ :

$$
d\left(\sigma(\mathcal{L}(\alpha, \theta, h)), h \sum_{j=0}^{J} \mu_{j, n} h^{j}\right) \leq C h^{J+2}
$$

and we have $\mu_{0, n}=\sigma(\theta), \mu_{1, n}=\nu_{n}\left(\mathfrak{S}_{\theta}\left(D_{\hat{\tau}}, \hat{\tau}\right)\right)$.

Main result The aim of this paper is to establish the following accurate estimate for $\lambda_{n}(h)$ :

Theorem 1.4 For all $\alpha>0, \theta \in\left(0, \frac{\pi}{2}\right)$ and $n \geq 1$, there exists a sequence $\left(\mu_{j, n}\right)_{j \geq 0}$ and $\varepsilon_{0}>0$ s. t. for $\left|x_{0}\right|+\left|y_{0}\right|+\left|z_{0}\right| \leq \varepsilon_{0}$,

$$
\lambda_{n}(h) \underset{h \rightarrow 0}{\sim} h \sum_{j \geq 0} \mu_{j, n} h^{j}
$$

and we have $\mu_{0, n}=\sigma(\theta), \mu_{1, n}=\nu_{n}\left(\boldsymbol{S}_{\theta}\left(D_{\hat{\tau}}, \hat{\tau}\right)\right)$. 
Organization of the paper The paper is organized as follows. In Section 2, we recall a first rough lower bound for $\lambda_{n}(h)$ and provide the corresponding normal Agmon estimates for the eigenfunctions. In particular, we will see that the first eigenfunctions are living in a neighborhood of the boundary of size $h^{1 / 2}$. In Section 3, we use these rough results to improve the localization of the eigenfunctions with respect to the variables $(\hat{s}, \hat{t})$. In Section 4, we use those localizations estimates to reduce the problem to the study of a normal form (similarly as in [17]) involving an harmonic oscillator operating in $\hat{\tau}$ to improve the approximation of the eigenfunctions in order to estimate the spectral gap between the eigenvalues and deduce Theorem 1.4.

\section{First lower bound and localization estimates}

In this section, we are concerned by Agmon estimates (with respect to $(x, y)$ ) satisfied by an eigenfunction $u_{h}$ associated to $\lambda_{n}(h)$.

\subsection{Agmon estimates}

We shall prove the following tangential localization:

Proposition 2.1 For all $\delta>0$, there exist $C>0$ and $h_{0}>0$ such that for $h \in\left(0, h_{0}\right)$ :

$$
\begin{gathered}
\int_{\Omega_{0}} e^{\delta\left(x^{2}+y^{2}\right) / h^{1 / 4}}\left|u_{h}\right|^{2} d x d y d z \leq C\left\|u_{h}\right\|^{2} \\
\int_{\Omega_{0}} e^{\delta\left(x^{2}+y^{2}\right) / h^{1 / 4}}\left\|\nabla u_{h}\right\|^{2} d x d y d z \leq C h^{-1}\left\|u_{h}\right\|^{2} .
\end{gathered}
$$

Before starting the proof, let us recall a rough lower bound for $\lambda_{1}(h)$ first obtained in [19] (see also [10, Theorem 9.1.1]):

Proposition 2.2 There exist $C>0$ and $h_{0}>0$ such that, for $h \in\left(0, h_{0}\right)$ :

$$
\lambda_{n}(h) \geq \sigma(\theta) h-C h^{5 / 4} .
$$

Combining the upper and lower bounds of $\lambda_{n}(h)$, this is standard to deduce the following normal Agmon estimates (see [16]):

Proposition 2.3 There exist $\delta>0, C>0$ and $h_{0}>0$ such that for all $h \in\left(0, h_{0}\right)$, we have :

$$
\int_{\Omega_{0}} e^{\delta h^{-1 / 2} z}\left(\left|u_{h}\right|^{2}+h^{-1}\left|(i h \nabla+\boldsymbol{A}) u_{h}\right|^{2}\right) d x d y d z \leq C\left\|u_{h}\right\|^{2}
$$

Let us now deal with the proof of Proposition 2.1. 
Proof of Proposition 2.1 We begin by writing the Agmon identity (see [1, 2]):

$$
\lambda_{n}(h)\left\|\exp (\Phi) u_{h}\right\|^{2}=q_{h}\left(\exp (\Phi) u_{h}\right)-h^{2}\left\||\nabla \Phi| \exp (\Phi) u_{h}\right\|^{2},
$$

for $\Phi$ a Lipschitzian function (depending on $h$ ) to be determined. We estimate $q_{h}\left(\exp (\Phi) u_{h}\right)$ thanks to a localization technique. We use the following partition of unity :

$$
\begin{aligned}
& \sum_{j} \chi_{j, h}^{2}=1, \\
& \sum_{j}\left\|\nabla \chi_{j, h}\right\|^{2} \leq C h^{-2 \rho},
\end{aligned}
$$

where the $\chi_{j, h}$ are smooth cutoff functions supported in balls of size $h^{\rho}$. The IMS formula (cf. [6]) provides:

$$
q_{h}\left(\exp (\Phi) u_{h}\right) \geq \sum_{j} q_{h}\left(\chi_{j, h} \exp (\Phi) u_{h}\right)-C h^{2-2 \rho} \sum_{j}\left\|\chi_{j, h} \exp (\Phi) u_{h}\right\|^{2} .
$$

We let: $\phi_{j, h}=\chi_{j, h} \exp (\Phi) u_{h}$. We now perform the approximation by the constant magnetic field case to give a lower bound for $q_{h}\left(\phi_{j, h}\right)$. We introduce the linear approximation of $A$ in the ball $B_{j}$ :

$A_{j}^{\operatorname{lin}}(x, y, z)=A\left(x_{j}, y_{j}, z_{j}\right)+2 \alpha z_{j} x_{j}\left(x-x_{j}\right)+\left(2 \alpha z_{j} y_{j}-\sin \theta\right)\left(y-y_{j}\right)+\left(\cos \theta+\alpha\left(x_{j}^{2}+y_{j}^{2}\right)\right)\left(z-z_{j}\right)$.

It satisfies:

$$
\left|A(x, y, z)-A_{j}^{\operatorname{lin}}(x, y, z)\right| \leq C\left(\left|x-x_{j}\right|^{2}+\left|y-y_{j}\right|^{2}+\left|z-z_{j}\right|^{2}\right) .
$$

Then, we have, with the Cauchy-Schwarz inequality, for all $\varepsilon>0$ :

$q_{h}\left(\phi_{j, h}\right) \geq(1-\varepsilon)\left(\left\|h D_{y} \phi_{j, h}\right\|^{2}+\left\|h D_{z} \phi_{j, h}\right\|^{2}+\left\|\left(h D_{x}+A_{j}^{\operatorname{lin}}\right) \phi_{j, h}\right\|^{2}\right)-\varepsilon^{-1} C h^{4 \rho}\left\|\phi_{j, h}\right\|^{2}$.

For the balls intersecting the boundary, we have:

$$
\left\|h D_{y} \phi_{j, h}\right\|^{2}+\left\|h D_{z} \phi_{j, h}\right\|^{2}+\left\|\left(h D_{x}+A_{j}^{\text {lin }}\right) \phi_{j, h}\right\|^{2} \geq \hat{\boldsymbol{\beta}}\left(x_{j}, y_{j}\right) h\left\|\phi_{j, h}\right\|^{2} \geq h\left\|\phi_{j, h}\right\|^{2} \sigma(\theta)
$$

and for the other balls:

$$
\left\|h D_{y} \phi_{j, h}\right\|^{2}+\left\|h D_{z} \phi_{j, h}\right\|^{2}+\left\|\left(h D_{x}+A_{j}^{\operatorname{lin}}\right) \phi_{j, h}\right\|^{2} \geq\left\|\boldsymbol{\beta}\left(x_{j}, y_{j}\right)\right\| h\left\|\phi_{j, h}\right\|^{2} \geq h\left\|\phi_{j, h}\right\|^{2} \inf _{\Omega_{0}}\|\boldsymbol{\beta}\| .
$$

The optimization of $\varepsilon$ provides: $\varepsilon=h^{2 \rho-1 / 2}$ and the optimization of $\rho$ leads to: $\rho=\frac{3}{8}$. With this choice and (2.1), we get:

$$
\sum_{j}\left(q_{h}\left(\phi_{j, h}\right)-C h^{5 / 4}\left\|\phi_{j, h}\right\|^{2}-h^{2}\left\|\phi_{j, h} \nabla \Phi\right\|^{2}-\lambda_{1}(h)\left\|\phi_{j, h}\right\|^{2}\right) \leq 0 .
$$


We recall the rough upper bound (cf. Theorem 1.3):

$$
\lambda_{1}(h) \leq \sigma(\theta) h+C h^{2}
$$

We split the sum into two parts:

$$
\sum_{j}=\sum_{j}^{\text {bnd }}+\sum_{j}^{\text {int }}
$$

We choose $\Phi=\delta h^{-1 / 4}\left(x^{2}+y^{2}\right)$, so that, on the one hand, there exists $c>0$ (cf. (2.5)) such that:

$$
\sum_{j}^{\text {int }}\left(q_{h}\left(\phi_{j, h}\right)-C h^{5 / 4}\left\|\phi_{j, h}\right\|^{2}-h^{2}\left\|\phi_{j, h} \nabla \Phi\right\|^{2}-\lambda_{1}(h)\left\|\phi_{j, h}\right\|^{2}\right) \geq c h \sum_{j}^{\text {int }}\left\|\phi_{j, h}\right\|^{2} \geq 0
$$

and on the other hand (cf. (2.4)):

$$
\begin{aligned}
& \sum_{j}^{\text {bnd }}\left(q_{h}\left(\phi_{j, h}\right)-C h^{5 / 4}\left\|\phi_{j, h}\right\|^{2}-h^{2}\left\|\phi_{j, h} \nabla \Phi\right\|^{2}-\lambda_{1}(h)\left\|\phi_{j, h}\right\|^{2}\right) \\
& \left.\geq \sum_{j}^{\text {bnd }} \int_{\Omega_{0}}\left(\hat{\boldsymbol{\beta}}\left(x_{j}, y_{j}\right) h-\sigma(\theta) h-C(\delta) h^{5 / 4}\right)\right)\left|\phi_{j, h}\right|^{2} d x d y d z .
\end{aligned}
$$

Therefore, we deduce:

$$
\left.c h \sum_{j}^{\text {int }}\left\|\phi_{j, h}\right\|^{2}+\sum_{j}^{\text {bnd }} \int_{\Omega_{0}}\left(\hat{\boldsymbol{\beta}}\left(x_{j}, y_{j}\right) h-\sigma(\theta) h-C(\delta) h^{5 / 4}\right)\right)\left|\phi_{j, h}\right|^{2} d x d y d z \leq 0 .
$$

By non degeneracy of the minimum of $\hat{\boldsymbol{\beta}}$ (see (1.10)), we get the existence of $c_{1}>0$ and $c_{2}>0$ such that for all $j$ :

$$
c_{2}\left(x_{j}^{2}+y_{j}^{2}\right) \geq \hat{\boldsymbol{\beta}}\left(x_{j}, y_{j}\right)-\sigma(\theta) \geq c_{1}\left(x_{j}^{2}+y_{j}^{2}\right) .
$$

We now split the sum on the boundary into two parts: the $j$ 's such that $x_{j}^{2}+y_{j}^{2} \geq 2 h^{1 / 4} c_{1}^{-1} C(\delta)$ and the $j$ 's such that: $x_{j}^{2}+y_{j}^{2}<2 h^{1 / 4} c_{1}^{-1} C(\delta)$. We infer:

$$
c \sum_{j}^{\text {int }} \int_{\Omega_{0}}\left|\chi_{j, h} \exp (\Phi) u_{h}\right|^{2} d x d y d z+\sum_{j}^{\text {bnd }} \int_{\Omega_{0}}\left|\chi_{j, h} \exp (\Phi) u_{h}\right|^{2} d x d y d z \leq C\left\|u_{h}\right\|^{2} .
$$

Corollary 2.4 For all $\eta>0$, we have:

$$
\begin{gathered}
\int_{|x|+|y| \geq h^{1 / 8-\eta}}|x|^{k}|y|^{l}|z|^{m}\left(\left|u_{h}\right|^{2}+\left|D_{x} u_{h}\right|^{2}+\left|D_{y} u_{h}\right|^{2}+\left|D_{z} u_{h}\right|^{2}\right) d x d y d z=O\left(h^{\infty}\right)\left\|u_{h}\right\|^{2} . \\
\int_{z \geq h^{1 / 2-\eta}}|x|^{k}|y|^{l}|z|^{m}\left(\left|u_{h}\right|^{2}+\left|D_{x} u_{h}\right|^{2}+\left|D_{y} u_{h}\right|^{2}+\left|D_{z} u_{h}\right|^{2}\right) d x d y d z=O\left(h^{\infty}\right)\left\|u_{h}\right\|^{2} .
\end{gathered}
$$


Let us consider $\eta>0$ small enough and introduce the cutoff function defined by:

$$
\chi_{h}(x, y)=\chi_{0}\left(h^{-1 / 8+\eta} x, h^{-1 / 8+\eta} y, h^{-1 / 2+\eta} z\right),
$$

where $\chi_{0}$ is a smooth cutoff function being 1 near $(0,0,0)$. We can notice, by elliptic regularity, that $\chi_{h} u_{h}$ is smooth (as it is supported away from the vertices).

Let us consider $N \geq 1$. For $n=1, \cdots, N$, let us consider $u_{n, h}$ a $L^{2}$-normalized eigenfunction associated with $\lambda_{n}(h)$ so that $\left\langle u_{n, h}, u_{m, h}\right\rangle=0$ for $n \neq m$. We let:

$$
\mathfrak{E}_{N}(h)=\operatorname{span}_{n=1, \cdots, N} u_{n, h} .
$$

We notice that Propositions 2.3 and 2.1 hold for the elements of $\mathfrak{E}_{N}(h)$. As a consequence of Propositions 2.3 and 2.1, we have:

Corollary 2.5 We have:

$$
q_{h}\left(\tilde{u}_{h}\right) \leq \lambda_{N}(h)+O\left(h^{\infty}\right), \quad \text { with } \tilde{u}_{h}=\chi_{h} u_{h},
$$

where $u_{h} \in \mathfrak{E}_{N}(h)$.

Agmon estimates of higher order In the last paragraph we proved Agmon estimates for $u_{h}$ and its first derivatives. We will also need estimates for the higher derivatives. This is the aim of the following proposition (this kind of higher order estimates can be found for instance in [12]):

Proposition 2.6 For all $\nu \in \mathbb{N}^{3}$, there exists $\delta>0, \gamma \geq 0, h_{0}>0$ and $C>0$ such that, for $h \in\left(0, h_{0}\right)$ :

$$
\begin{gathered}
\int e^{\delta h^{-1 / 2} z}\left|D^{\nu} \tilde{u}_{h}\right|^{2} d x d y d z \leq C h^{-\gamma}\left\|\tilde{u}_{h}\right\|^{2} . \\
\int e^{\delta h^{-1 / 4}\left(x^{2}+y^{2}\right)}\left|D^{\nu} \tilde{u}_{h}\right|^{2} d x d y d z \leq C h^{-\gamma}\left\|\tilde{u}_{h}\right\|^{2},
\end{gathered}
$$

where $u_{h} \in \mathfrak{E}_{N}(h)$.

Proof: For $|\nu|=1$, this is already proved. Let $\nu$ such that $|\nu| \geq 1$ and assume that the inequality is proved for all multi-indices with length smaller than $|\nu|$. We recall that, when $u_{h}$ is an eigenfunction:

$$
\mathcal{L}(h) \tilde{u}_{h}=\lambda_{n}(h) \tilde{u}_{h}+\left[\mathcal{L}(h), \chi_{h}\right] u_{h} .
$$

Let us apply $D^{\nu}$ to the equation. We get:

$$
\mathcal{L}(h) D^{\nu} \tilde{u}_{h}=\lambda_{h}(h) D^{\nu} \tilde{u}_{h}+D^{\nu}\left[\mathcal{L}(h), \chi_{h}\right] u_{h}+\left[\mathcal{L}(h), D^{\nu}\right] \tilde{u}_{h} .
$$


It follows, for $\Phi=\delta h^{-1 / 2} z$ or $\Phi=\delta h^{-1 / 4}\left(x^{2}+y^{2}\right)$ :

$\mathcal{L}(h) e^{\Phi} D^{\nu} \tilde{u}_{h}=\lambda_{n}(h) e^{\Phi} D^{\nu} \tilde{u}_{h}+e^{\Phi} D^{\nu}\left[\mathcal{L}(h), \chi_{h}\right] u_{h}+e^{\Phi}\left[\mathcal{L}(h), D^{\nu}\right] \tilde{u}_{h}+\left[\mathcal{L}(h), e^{\Phi}\right] D^{\nu} \tilde{u}_{h}$.

Then, we take the scalar product with $e^{\Phi} D^{\nu} \tilde{u}_{h}$. From the recursion assumption and up to choose $\delta$ smaller to absorbe polynomials, we deduce the existence of $\gamma_{1}>0$ and $\gamma_{2}>0$ such that:

$$
\begin{aligned}
& \left\langle\left[\mathcal{L}(h), e^{\Phi}\right] D^{\nu} \tilde{u}_{h}, e^{\Phi} D^{\nu} \tilde{u}_{h}\right\rangle=h^{2}\left\|\nabla \Phi e^{\Phi} D^{\nu} \tilde{u}_{h}\right\|^{2} \leq C h^{-\gamma_{1}}\left\|\tilde{u}_{h}\right\|^{2}, \\
& \left|\left\langle e^{\Phi}\left[\mathcal{L}(h), D^{\nu}\right] \tilde{u}_{h}, e^{\Phi} D^{\nu} \tilde{u}_{h}\right\rangle\right| \leq C h^{-\gamma_{1}}\left\|\tilde{u}_{h}\right\|^{2}, \\
& \left|\left\langle e^{\Phi} D^{\nu}\left[\mathcal{L}(h), \chi_{h}\right] u_{h}, e^{\Phi} D^{\nu} \tilde{u}_{h}\right\rangle\right| \leq C h^{-\gamma_{1}}\left\|\tilde{u}_{h}\right\|^{2}+C h^{\gamma_{2}} q_{h}\left(e^{\Phi} D^{\nu} \tilde{u}_{h}\right) .
\end{aligned}
$$

We infer:

$$
q_{h}\left(e^{\Phi} D^{\nu} \tilde{u}_{h}\right) \leq C h^{-\gamma_{1}}\left\|\tilde{u}_{h}\right\|^{2} .
$$

In particular, we get the control of $D_{t} D^{\nu}, D_{s} D^{\nu}$ and, up to loose again some negative power of $h$, we get the control of $D_{r} D^{\nu}$. The extension to $u_{h} \in \mathfrak{E}_{N}(h)$ is then standard.

In particular, we infer:

Corollary 2.7 For all $\eta>0$, we have, for all $\nu \in \mathbb{N}^{3}$ :

$$
\begin{gathered}
\int_{|x|+|y| \geq h^{1 / 8-\eta}}|x|^{k}|y|^{l}|z|^{m}\left|D^{\nu} \tilde{u}_{h}\right|^{2} d x d y d z=O\left(h^{\infty}\right)\left\|\tilde{u}_{h}\right\|^{2}, \\
\int_{z \geq h^{1 / 2-\eta}}|x|^{k}|y|^{l}|z|^{m}\left|D^{\nu} \tilde{u}_{h}\right|^{2} d x d y d z=O\left(h^{\infty}\right)\left\|\tilde{u}_{h}\right\|^{2}
\end{gathered}
$$

where $u_{h} \in \mathfrak{E}_{N}(h)$.

\subsection{Toward a normal form}

For $u_{h} \in \mathfrak{E}_{N}(h)$, we let:

$$
w_{h}(r, s, t)=\chi_{h}^{\text {resc }}(r, s, t) u_{h}^{\text {resc }}(r, s, t)=\chi_{0}\left(h^{3 / 8+\eta} r, h^{3 / 8+\eta} s, h^{\eta} t\right) u_{h}\left(h^{1 / 2} r, h^{1 / 2} s, h^{1 / 2} t\right)
$$

and

$$
v_{h}(\hat{\tau}, \hat{s}, \hat{t})=U_{\theta} \mathcal{F} w_{h} .
$$

We consider $\mathfrak{F}_{N}(h)$ the image of $\mathfrak{E}_{N}(h)$ by these transformations. We can reformulate Corollary 2.5.

Corollary 2.8 With the previous notation, we have the lower bound, for $v_{h} \in \mathfrak{F}_{N}(h)$ :

$$
q_{h}^{\text {Normal }}\left(v_{h}\right) \leq \lambda_{N}^{\text {resc }}(h)+O\left(h^{\infty}\right),
$$

where $\lambda_{N}^{\text {resc }}(h)=h^{-1} \lambda_{N}(h)$. 
We can also notice that, when $u_{h}$ is an eigenfunction associated with $\lambda_{p}(h)$ :

$$
\mathcal{L}^{\text {Normal }}(h) v_{h}=\lambda_{p}^{\text {resc }}(h) v_{h}+r_{h},
$$

where the remainder $r_{h}$ is $O\left(h^{\infty}\right)$ in the sense of Corollary 2.7.

In the following, we aim at proving localization and approximation estimates for $v_{h}$ rather that $u_{h}$. Moreover, these approximations will allow us to estimate the energy $q_{h}^{\text {Normal }}\left(v_{h}\right)$.

\section{Approximation and refined localizations in the phase space}

This section aims at estimating momenta of $v_{h}$ with respect to polynoms in the phase space. Before starting the analysis, let us recall the link (cf. (1.6)) between the variables $(\tau, s, t)$ and $(\hat{\tau}, \hat{s}, \hat{t})$ :

$$
D_{\hat{\tau}}=D_{\tau}+\frac{1}{\sin \theta} D_{s}, \quad D_{\hat{s}}=D_{s}, \quad D_{\hat{t}}=D_{t} .
$$

We will use the following obvious remark:

Remark 3.1 We can notice that if $\phi$ is supported in $\operatorname{supp}\left(\chi_{h}\right)$, we have:

$$
q_{h}^{\text {resc }}(\phi) \geq(1-\varepsilon) \mathcal{Q}_{\theta}(\phi)-C h^{1 / 2-6 \eta} \varepsilon^{-1}\|\phi\|^{2} .
$$

Optimizing in $\varepsilon$, we have:

$$
q_{h}^{\text {resc }}(\phi) \geq\left(1-h^{1 / 4-3 \eta}\right) \mathcal{Q}_{\theta}(\phi)-C h^{1 / 4-3 \eta}\|\phi\|^{2} .
$$

Moreover, when the support of $\phi$ avoids the boundary, we have:

$$
\mathcal{Q}_{\theta}(\phi) \geq\|\phi\|^{2}
$$

\subsection{Localizations in $\hat{s}$ and $\hat{t}$}

In this subsection, many localizations lemmas with respect to $\hat{s}$ and $\hat{t}$ are proved.

Estimates with respect to $\hat{s}$ and $\hat{t}$ We begin to prove estimates depending only on the variables $\hat{s}$ and $\hat{t}$.

Lemma 3.2 Let $N \geq 1$. For all $k, n$, there exist $h_{0}>0$ and $C(k, n)>0$ such that, for all $h \in\left(0, h_{0}\right)$ :

$$
\begin{aligned}
& \left\|\hat{t}^{k} \hat{s}^{n+1} v_{h}\right\| \leq C(k, n)\left\|v_{h}\right\|, \\
& \left\|\hat{t}^{k} D_{\hat{s}}\left(\hat{s}^{n} v_{h}\right)\right\| \leq C(k, n)\left\|v_{h}\right\| \\
& \left\|\hat{t}^{k} D_{\hat{t}}\left(\hat{s}^{n} v_{h}\right)\right\| \leq C(k, n)\left\|v_{h}\right\| .
\end{aligned}
$$

for $v_{h} \in \mathfrak{F}_{N}(h)$. 
Proof: We prove the estimates when $v_{h}$ is the image of an eigenfunction associated to $\lambda_{p}(h)$ with $p=1, \cdots, N$.

Case $n=0$ Let us analyze the case $n=0$. (3.4) follows from the normal Agmon estimates. We have:

$$
q_{h}^{\text {Normal }}\left(\hat{t}^{k} v_{h}\right) \leq \lambda_{p}^{\text {resc }}\left\|\hat{t}^{k} v_{h}\right\|^{2}+\left|\left\langle\left[D_{\hat{t}}^{2}, \hat{t}^{k}\right] w_{h}, \hat{t}^{k} v_{h}\right\rangle\right|+O\left(h^{\infty}\right)\left\|v_{h}\right\|^{2} .
$$

The normal Agmon estimates provide:

$$
\left|\left\langle\left[D_{\hat{t}}^{2}, \hat{t}^{k}\right] v_{h}, \hat{t}^{k} v_{h}\right\rangle\right| \leq C\left\|v_{h}\right\|^{2}
$$

and thus:

$$
q_{h}^{\text {Normal }}\left(\hat{t}^{k} v_{h}\right) \leq C\left\|v_{h}\right\|^{2} .
$$

We deduce (3.3). We also have:

$$
\left\|\hat{t}^{k}\left(-\hat{s} \sin \theta+\hat{t} \cos \theta+R_{h}\right) v_{h}\right\|^{2} \leq C\left\|v_{h}\right\|^{2}
$$

We use the basic lower bound:

$$
\left\|\hat{t}^{k}\left(-\hat{s} \sin \theta+\hat{t} \cos \theta+R_{h}\right) v_{h}\right\|^{2} \geq \frac{1}{2}\left\|\hat{t}^{k} \hat{s} \sin \theta v_{h}\right\|^{2}-2\left\|\left(\hat{t}^{k+1} \cos \theta+\hat{t}^{k} R_{h}\right) v_{h}\right\|^{2} .
$$

Moreover, we have (using the support of $\chi_{h}^{\text {resc }}$ ):

$$
\left\|\hat{t}^{k} R_{h} v_{h}\right\| \leq C h\left(h^{-3 / 8-\eta}\right)^{2}\left\|\hat{t}^{k+1} v_{h}\right\| \leq C h\left(h^{-3 / 8-\eta}\right)^{2}\left\|v_{h}\right\|,
$$

the last inequality coming from the normal Agmon estimates. Thus, we get:

$$
\left\|\hat{t}^{k} \hat{s} v_{h}\right\|^{2} \leq C\left\|v_{h}\right\|^{2}
$$

Recursion We apply $\hat{t}^{k} \hat{s}^{n+1}$ to (2.7), take the scalar product with $t^{k} \hat{s}^{n+1} v_{h}$ and it follows:

$$
\begin{aligned}
q_{h}^{\text {Normal }}\left(\hat{t}^{k} \hat{s}^{n+1} v_{h}\right) & \leq \lambda_{p}^{\text {resc }}(h)\left\|\hat{t}^{k} \hat{s}^{n+1} v_{h}\right\|^{2}+C\left\|\hat{t}^{k-2} \hat{s}^{n+1} v_{h}\right\|\left\|\hat{t}^{k} \hat{s}^{n+1} v_{h}\right\| \\
& +C\left\|\hat{t}^{k-1} D_{\hat{t}} \hat{s}^{n} v_{h}\right\|\left\|\hat{t}^{k} \hat{s}^{n+1} v_{h}\right\|+C\left\|\hat{t}^{k} D_{\hat{s}} \hat{s}^{n} w_{h}\right\|\left\|\hat{t}^{k} \hat{s}^{n+1} v_{h}\right\| \\
& +C\left\|\hat{t}^{k} \hat{s}^{n-1} v_{h}\right\|\left\|\hat{t}^{k} \hat{s}^{n+1} v_{h}\right\|+\left|\left\langle\hat{t}^{k}\left[\hat{s}^{n+1},\left(-\hat{s} \sin \theta+\hat{t} \cos \theta+R_{h}\right)^{2}\right] v_{h}, \hat{t}^{k} \hat{s}^{n+1}\right\rangle\right|,
\end{aligned}
$$

where

$$
R_{h}=h \alpha \hat{t}\left\{\left(D_{\hat{\tau}}-(\sin \theta)^{-1} D_{\hat{s}}\right)^{2}+\left(\hat{s}+(\sin \theta)^{-1} \hat{\tau}\right)^{2}\right\}
$$

We have:

$$
\begin{aligned}
& {\left[\hat{s}^{n+1},\left(-\hat{s} \sin \theta+\hat{t} \cos \theta+R_{h}\right)^{2}\right]} \\
& =\left[\hat{s}^{n+1}, R_{h}\right]\left(-\hat{s} \sin \theta+\hat{t} \cos \theta+R_{h}\right)+\left(-\hat{s} \sin \theta+\hat{t} \cos \theta+R_{h}\right)\left[\hat{s}^{n+1}, R_{h}\right] .
\end{aligned}
$$


Let us analyze the commutator $\left[\hat{s}^{n+1}, R_{h}\right]$. We can write:

$$
\left[\hat{s}^{n+1}, R_{h}\right]=\alpha h \hat{t}\left[\hat{s}^{n+1},\left(D_{\hat{\tau}}-(\sin \theta)^{-1} D_{\hat{s}}\right)^{2}\right]
$$

and:

$$
\begin{aligned}
{\left[\left(D_{\hat{\tau}}-(\sin \theta)^{-1} D_{\hat{s}}\right)^{2}, \hat{s}^{n+1}\right] } & =(\sin \theta)^{-2} n(n+1) \hat{s}^{n-1} \\
& +2 i(\sin \theta)^{-1}(n+1)\left(D_{\hat{\tau}}-(\sin \theta)^{-1} D_{\hat{s}}\right) \hat{s}^{n}
\end{aligned}
$$

we infer:

$$
\begin{aligned}
& {\left[\hat{s}^{n+1},\left(-\hat{s} \sin \theta+\hat{t} \cos \theta+R_{h}\right)^{2}\right]} \\
& =\left(\alpha h \hat{t}(\sin \theta)^{-2} n(n+1) \hat{s}^{n-1}+2 i \alpha h \hat{t}(\sin \theta)^{-1}(n+1)\left(D_{\hat{\tau}}-(\sin \theta)^{-1} D_{\hat{s}}\right) \hat{s}^{n}\right)\left(V_{\theta}+R_{h}\right) \\
& +\left(V_{\theta}+R_{h}\right)\left(\alpha h \hat{t}(\sin \theta)^{-2} n(n+1) \hat{s}^{n-1}+2 i \alpha h \hat{t}(\sin \theta)^{-1}(n+1)\left(D_{\hat{\tau}}-(\sin \theta)^{-1} D_{\hat{s}}\right) \hat{s}^{n}\right)
\end{aligned}
$$

After having computed a few more commutators, the terms of $\left[\hat{s}^{n+1},\left(-\hat{s} \sin \theta+\hat{t} \cos \theta+R_{h}\right)^{2}\right]$ are in the form:

$$
\begin{gathered}
\hat{t}^{l} \hat{s}^{m}, h \hat{t}^{l}\left(D_{\hat{\tau}}-(\sin \theta)^{-1} D_{\hat{s}}\right) \hat{s}^{m}, h^{2} \hat{t}^{l}\left(D_{\hat{\tau}}-(\sin \theta)^{-1} D_{\hat{s}}\right)^{3} \hat{s}^{m}, \\
h^{2} \hat{t}^{l}\left(\hat{s}+(\sin \theta)^{-1} \hat{\tau}\right)^{2}\left(D_{\hat{\tau}}+(\sin \theta)^{-1} D_{\hat{s}}\right) \hat{s}^{m}
\end{gathered}
$$

with $m \leq n+1$ and $l=0,1,2$.

Let us examine for instance the term $h^{2} \hat{t}^{l}\left(\hat{s}+(\sin \theta)^{-1} \hat{\tau}\right)^{2}\left(D_{\hat{\tau}}+(\sin \theta)^{-1} D_{\hat{s}}\right) \hat{s}^{m}$. We have, after the inverse Fourier transform and translation:

$$
h^{2}\left\|\hat{t}^{l}\left(\hat{s}+(\sin \theta)^{-1} \hat{\tau}\right)^{2}\left(D_{\hat{\tau}}+(\sin \theta)^{-1} D_{\hat{s}}\right) \hat{s}^{m} v_{h}\right\| \leq C h^{2}\left(h^{-3 / 8-\eta}\right)^{3}\left\|\hat{t}^{l} \hat{s}^{m} v_{h}\right\|
$$

where we have used the support of $\chi_{h}^{\text {resc }}($ see (2.6)). We get:

$$
\left|\left\langle\hat{t}^{k}\left[\hat{s}^{n+1},\left(-\hat{s} \sin \theta+\hat{t} \cos \theta+R_{h}\right)^{2}\right] v_{h}, \hat{t}^{k} \hat{s}^{n+1} v_{h}\right\rangle\right| \leq C\left\|\hat{t}^{k} \hat{s}^{n+1} v_{h}\right\| \sum_{j=0}^{n+1} \sum_{l=0}^{k+2}\left\|\hat{t}^{l} \hat{s}^{j} v_{h}\right\| .
$$

We deduce by the recursion assumption:

$$
q_{h}^{\text {Normal }}\left(\hat{t}^{k} \hat{s}^{n+1} v_{h}\right) \leq C\left\|v_{h}\right\|^{2} .
$$

We infer that, for all $k$ :

$$
\left\|D_{\hat{t}}\left(\hat{t}^{k} \hat{s}^{n+1}\right) v_{h}\right\| \leq C\left\|v_{h}\right\| \text { and }\left\|D_{\hat{s}}\left(\hat{t}^{k} \hat{s}^{n+1}\right) v_{h}\right\| \leq C\left\|v_{h}\right\| .
$$

Moreover, we also deduce:

$$
\left\|\left(V_{\theta}+R_{h}\right) \hat{t}^{k} \hat{s}^{n+1} v_{h}\right\| \leq C\left\|v_{h}\right\|
$$

from which we find:

$$
\left\|\hat{t}^{k} \hat{s}^{n+2} v_{h}\right\| \leq C\left\|v_{h}\right\|
$$

We will also need a control of the derivatives with respect to $\hat{s}$. 
Lemma 3.3 For all $m, n, k$, there exist $h_{0}>0$ and $C(m, n, k)>0$ such that for $h \in\left(0, h_{0}\right)$ :

$$
\begin{aligned}
& \left\|\hat{t}^{k} D_{\hat{s}}^{m+1} \hat{s}^{n} v_{h}\right\| \leq C(k, m, n)\left\|v_{h}\right\| \\
& \left\|\hat{t}^{k} D_{\hat{s}}^{m} D_{\hat{t}} \hat{s}^{n} v_{h}\right\| \leq C(k, m, n)\left\|v_{h}\right\|,
\end{aligned}
$$

for $v_{h} \in \mathfrak{F}_{N}(h)$.

Proof: For $m=0$, this is done in Lemma 3.2.

Let us assume that the assumptions are true for all $l \leq m$ and all $k$ and $n$. We apply $\hat{t}^{k} D_{\hat{s}}^{m+1} \hat{s}^{n}$ to (2.7) and take the scalar product with $\hat{t}^{k} D_{\hat{s}}^{m+1} \hat{s}^{n} v_{h}$. We shall estimate commutators. We get:

$$
\begin{aligned}
& \left|\left\langle\left[D_{\hat{t}}^{2}, \hat{t}^{k} D_{\hat{s}}^{m+1} \hat{s}^{n}\right] v_{h}, \hat{t}^{k} D_{\hat{s}}^{m+1} \hat{s}^{n} v_{h}\right\rangle\right| \\
& \leq C\left\|\hat{t}^{k} D_{\hat{t}} D_{\hat{s}}^{m+1} \hat{s}^{n} v_{h}\right\|\left\|\hat{t}^{k-1} D_{\hat{s}}^{m+1} \hat{s}^{n} v_{h}\right\|+C\left\|\hat{t}^{k-2} D_{\hat{s}}^{m+1} \hat{s}^{n} v_{h}\right\|\left\|\hat{t}^{k} D_{\hat{s}}^{m+1} \hat{s}^{n} v_{h}\right\| \\
& \leq C\left\|D_{\hat{t}} \hat{t}^{k} D_{\hat{s}}^{m+1} \hat{s}^{n} v_{h}\right\|\left\|\hat{t}^{k-1} D_{\hat{s}}^{m+1} \hat{s}^{n} v_{h}\right\|+C\left\|\hat{t}^{k-2} D_{\hat{s}}^{m+1} \hat{s}^{n} v_{h}\right\|\left\|\hat{t}^{k} D_{\hat{s}}^{m+1} \hat{s}^{n} v_{h}\right\| \\
& +C\left\|\hat{t}^{k-1} D_{\hat{s}}^{m+1} \hat{s}^{n} v_{h}\right\|^{2}
\end{aligned}
$$

and we deduce, with the recursion assumption, for all $\varepsilon>0$, the existence of $C_{\varepsilon}>0$ such that:

$$
\left|\left\langle\left[D_{\hat{t}}^{2}, \hat{t}^{k} D_{\hat{s}}^{m+1} \hat{s}^{n}\right] v_{h}, \hat{t}^{k} D_{\hat{s}}^{m+1} \hat{s}^{n} v_{h}\right\rangle\right| \leq C(k, m, n) \varepsilon q_{h}^{\text {Normal }}\left(\hat{t}^{k} D_{\hat{s}}^{m+1} \hat{s}^{n} v_{h}\right)+C_{\varepsilon}\left\|v_{h}\right\|^{2} .
$$

Moreover, we have in the same way:

$$
\left|\left\langle\left[D_{\hat{s}}^{2}, \hat{t}^{k} D_{\hat{s}}^{m+1} \hat{s}^{n}\right] v_{h}, \hat{t}^{k} D_{\hat{s}}^{m+1} \hat{s}^{n} v_{h}\right\rangle\right| \leq C(k, m, n) \varepsilon q_{h}^{\text {Normal }}\left(\hat{t}^{k} D_{\hat{s}}^{m+1} \hat{s}^{n} v_{h}\right)+C_{\varepsilon}\left\|v_{h}\right\|^{2} .
$$

Finally, we shall analyze:

$$
\left[\left(V_{\theta}(\hat{s}, \hat{t})+R_{h}\right)^{2}, \hat{t}^{k} D_{\hat{s}}^{m+1} \hat{s}^{n}\right]
$$

We have:

$$
\begin{aligned}
& {\left[\left(V_{\theta}(\hat{s}, \hat{t})+R_{h}\right)^{2}, D_{\hat{s}}^{m+1} \hat{s}^{n}\right]} \\
& =\left(V_{\theta}(\hat{s}, \hat{t})+R_{h}\right)\left[\left(V_{\theta}(\hat{s}, \hat{t})+R_{h}\right), D_{\hat{s}}^{m+1} \hat{s}^{n}\right]+\left[\left(V_{\theta}(\hat{s}, \hat{t})+R_{h}\right), D_{\hat{s}}^{m+1} \hat{s}^{n}\right]\left(V_{\theta}(\hat{s}, \hat{t})+R_{h}\right)
\end{aligned}
$$

The worst term is $h \hat{t}\left[\left(D_{\hat{s}}-(\sin \theta)^{-1} D_{\hat{\tau}}\right)^{2}, D_{\hat{s}}^{m+1} \hat{s}^{n}\right]$ but it can be dealt by using the support of $w_{h}$ (see (2.6) and the proof of Lemma 3.2). We deduce:

$$
\left\langle\mid\left[\left(V_{\theta}(\hat{s}, \hat{t})+R_{h}\right)^{2}, \hat{t}^{k} D_{\hat{s}}^{m+1} \hat{s}^{n}\right] v_{h}, \hat{t}^{k} D_{\hat{s}}^{m+1} \hat{s}^{n} v_{h}\right\rangle \mid \leq C(k, m, n)\left\|v_{h}\right\|^{2} .
$$

We infer the existence of $C(k, m, n)>0$ such that:

$$
(1-C(k, m, n) \varepsilon) q_{h}^{\text {Normal }}\left(\hat{t}^{k} D_{\hat{s}}^{m+1} \hat{s}^{n}\right) \leq C(k, m, n)\left\|v_{h}\right\|^{2} .
$$

and the conclusion follows by choosing $\varepsilon$ small enough..

In the next paragraph, we establish partial Agmon estimates with respect to $\hat{s}$ and $\hat{t}$. Roughly speaking, we can write the previous lemmas with $\hat{\tau} v_{h}$ and $D_{\hat{\tau}} v_{h}$ instead of $v_{h}$. 
Partial estimates involving $\hat{\tau}$ We now aim at adding $\hat{\tau}$ and $D_{\hat{\tau}}$ in the previous estimates. For instance, let us begin to prove that:

Lemma 3.4 For all $k \geq 0$, there exist $h_{0}>0$ and $C(k)>0$ such that, for $h \in\left(0, h_{0}\right)$ :

$$
\begin{aligned}
& \left\|\hat{t}^{k} \hat{\tau} v_{h}\right\| \leq C\left(\left\|\hat{\tau} v_{h}\right\|+\left\|v_{h}\right\|\right),\left\|\hat{t}^{k} D_{\hat{t}} \hat{\tau} v_{h}\right\| \leq C\left(\left\|\hat{\tau} v_{h}\right\|+\left\|v_{h}\right\|\right), \\
& \text { and }\left\|\hat{t}^{k} D_{\hat{s}} \hat{\tau} v_{h}\right\| \leq C\left(\left\|\hat{\tau} v_{h}\right\|+\left\|v_{h}\right\|\right)
\end{aligned}
$$

for $v_{h} \in \mathfrak{F}_{N}(h)$.

Proof: For $k=0$, we multiply (2.7) by $\hat{\tau}$ and take the scalar product with $\hat{\tau} v_{h}$. There is only one commutator to analyze:

$$
\left[\left(V_{\theta}+R_{h}\right)^{2}, \hat{\tau}\right]=\left[\left(V_{\theta}+R_{h}\right), \hat{\tau}\right]\left(V_{\theta}+R_{h}\right)+\left(V_{\theta}+R_{h}\right)\left[\left(V_{\theta}+R_{h}\right), \hat{\tau}\right]
$$

so that:

$$
\left[\left(V_{\theta}+R_{h}\right)^{2}, \hat{\tau}\right]=\left[R_{h}, \hat{\tau}\right]\left(V_{\theta}+R_{h}\right)+\left(V_{\theta}+R_{h}\right)\left[R_{h}, \hat{\tau}\right] .
$$

We deduce, thanks to the support of $w_{h}$ :

$$
\left|\left\langle\left[\left(V_{\theta}+R_{h}\right)^{2}, \hat{\tau}\right] v_{h}, \hat{\tau} v_{h}\right\rangle\right| \leq C\left\|v_{h}\right\|\left\|\hat{\tau} v_{h}\right\| \leq C\left(\left\|\hat{\tau} v_{h}\right\|^{2}+\left\|v_{h}\right\|^{2}\right)
$$

and we infer:

$$
q_{h}^{\text {Normal }}\left(\hat{\tau} v_{h}\right) \leq C\left(\left\|\hat{\tau} v_{h}\right\|^{2}+\left\|v_{h}\right\|^{2}\right)
$$

We get:

$$
\left\|D_{\hat{t}} \hat{\tau} v_{h}\right\| \leq C\left(\left\|\hat{\tau} v_{h}\right\|+\left\|v_{h}\right\|\right) \text { and }\left\|D_{\hat{s}} \hat{\tau} v_{h}\right\| \leq C\left(\left\|\hat{\tau} v_{h}\right\|+\left\|v_{h}\right\|\right)
$$

Recursion We assume that the property is true for all $0 \leq l \leq k$. We now multiply (2.7) by $\hat{t}^{k+1} \hat{\tau}$ and take the scalar product with $\hat{t}^{k+1} \hat{\tau} v_{h}$. We have:

$$
\begin{aligned}
& \left|\left\langle\left[D_{\hat{t}}^{2}, \hat{t}^{k+1} \hat{\tau}\right] v_{h}, \hat{t}^{k+1} \hat{\tau} v_{h}\right\rangle\right| \leq C\left(\left\|\hat{t}^{k} D_{\hat{t}} \hat{\tau} v_{h}\right\|+\left\|\hat{t}^{k-1} \hat{\tau} v_{h}\right\|\right)\left\|t^{k+1} \hat{\tau} v_{h}\right\| \\
& \leq C \varepsilon\left\|\hat{t}^{k+1} \hat{\tau} v_{h}\right\|^{2}+C_{\varepsilon}\left(\left\|\hat{\tau} v_{h}\right\|^{2}+\left\|v_{h}\right\|^{2}\right) .
\end{aligned}
$$

We have:

$$
\left|\left\langle\hat{t}^{k+1}\left[\left(V_{\theta}+R_{h}\right)^{2}, \hat{\tau}\right] v_{h}, \hat{t}^{k+1} \hat{\tau} v_{h}\right\rangle\right| \leq C \varepsilon q_{h}^{\text {Normal }}\left(\hat{t}^{k+1} \hat{\tau} v_{h}\right)+C_{\varepsilon}\left\|\hat{t}^{k+1} \hat{\tau} v_{h}\right\|^{2} .
$$

We deduce:

$$
(1-C \varepsilon) q_{h}^{\text {Normal }}\left(\hat{t}^{k+1} \hat{\tau} v_{h}\right) \leq(\sigma(\theta)+C \varepsilon)\left\|\hat{t}^{k+1} \hat{\tau} v_{h}\right\|^{2}+C\left\|\hat{\tau} v_{h}\right\|^{2}+C\left\|v_{h}\right\|^{2}
$$

It remains to apply Remark 3.1 and to use $\sigma(\theta)<1$ to deduce the control of $\hat{t}^{k+1} \hat{\tau} v_{h}$ and thus the one of $\hat{t}^{k+1} D_{\hat{t}}$ and $\hat{t}^{k+1} D_{\hat{s}}$.

As an easy consequence of the analysis of Lemma 3.4, we have: 
Lemma 3.5 For all $k \geq 0$, there exist $h_{0}>0$ and $C(k)>0$ such that, for $h \in\left(0, h_{0}\right)$ :

$$
\left\|\hat{t}^{k} \hat{s} \hat{\tau} v_{h}\right\| \leq C(k)\left(\left\|\hat{\tau} v_{h}\right\|+\left\|v_{h}\right\|\right)
$$

for $v_{h} \in \mathfrak{F}_{N}(h)$.

We can now prove the following lemma:

Lemma 3.6 For all $k, n$, there exist $h_{0}>0$ and $C(k, n)>0$ such that, for all $h \in\left(0, h_{0}\right)$ :

$$
\begin{aligned}
& \left\|\hat{\tau} t^{k} \hat{s}^{n+1} v_{h}\right\| \leq C(k, n)\left(\left\|\hat{\tau} v_{h}\right\|+\left\|v_{h}\right\|\right), \\
& \left\|\hat{\tau} t^{k} D_{\hat{s}}\left(\hat{s}^{n} v_{h}\right)\right\| \leq C(k, n)\left(\left\|\hat{\tau} v_{h}\right\|+\left\|v_{h}\right\|\right) \\
& \left\|\hat{\tau} t^{k} D_{\hat{t}}\left(\hat{s}^{n} v_{h}\right)\right\| \leq C(k, n)\left(\left\|\hat{\tau} v_{h}\right\|+\left\|v_{h}\right\|\right),
\end{aligned}
$$

for $v_{h} \in \mathfrak{F}_{N}(h)$.

Proof: The proof is based on a recursion with respect to $n$. For $n=0$, this is a consequence of Lemmas 3.4 and 3.5. We refer to the proof of Lemma 3.2. The only difference appears in the computation of the commutator $\left[\left(V_{\theta}+R_{h}\right)^{2}, \hat{\tau} \hat{s}^{n+1}\right]$. Indeed, $\left[\left(D_{\hat{\tau}}-(\sin \theta)^{-1} D_{\hat{s}}\right)^{2}, \hat{\tau} \hat{s}^{n+1}\right]$ provides an additional term in $\hat{s}^{n+1}$ but this one is controlled by Lemma 3.2 .

From this lemma, we deduce a stronger control with respect to the derivative with respect to $\hat{s}$ :

Lemma 3.7 For all $m, n, k$, there exist $h_{0}>0$ and $C(m, n, k)>0$ such that for $h \in\left(0, h_{0}\right)$ :

$$
\begin{aligned}
& \left\|\hat{\tau} \hat{t}^{k} D_{\hat{s}}^{m+1} \hat{s}^{n} v_{h}\right\| \leq C(k, m, n)\left(\left\|\hat{\tau} v_{h}\right\|+\left\|v_{h}\right\|\right), \\
& \left\|\hat{\tau} \hat{t}^{k} D_{\hat{s}}^{m} D_{\hat{t}} \hat{s}^{n} v_{h}\right\| \leq C(k, m, n)\left(\left\|\hat{\tau} v_{h}\right\|+\left\|v_{h}\right\|\right),
\end{aligned}
$$

for $v_{h} \in \mathfrak{F}_{N}(h)$

Proof: The proof can be done by recursion on $m$. The case $m=0$ comes from the previous lemma. Then, the recursion is the same as for the proof of Lemma 3.3 and uses Lemma 3.3 to control the additional commutators.

By the same arguments (symmetry between $\hat{\tau}$ and $D_{\hat{\tau}}$ ), we have:

Lemma 3.8 For all $m, n, k$, there exist $h_{0}>0$ and $C(m, n, k)>0$ such that for $h \in\left(0, h_{0}\right)$ :

$$
\begin{aligned}
& \left\|D_{\hat{\tau}} \hat{t}^{k} D_{\hat{s}}^{m+1} \hat{s}^{n} v_{h}\right\| \leq C(k, m, n)\left(\left\|D_{\hat{\tau}} v_{h}\right\|+\left\|v_{h}\right\|\right), \\
& \left\|D_{\hat{\tau}} \hat{t}^{k} D_{\hat{s}}^{m} D_{\hat{t}} \hat{s}^{n} v_{h}\right\| \leq C(k, m, n)\left(\left\|D_{\hat{\tau}} v_{h}\right\|+\left\|v_{h}\right\|\right),
\end{aligned}
$$

for $v_{h} \in \mathfrak{F}_{N}(h)$.

In the next subsection, we prove that $v_{h}$ behaves like $u_{\theta}(\hat{s}, \hat{t})$ with respect to $\hat{s}$ and $\hat{t}$. 


\subsection{Approximation of $v_{h}$}

Let us state the approximation result of this subsection:

Proposition 3.9 There exists $C>0$ and $h_{0}>0$ such that, if $0<h \leq h_{0}$ and $v_{h} \in \mathfrak{F}_{N}(h)$ :

$$
\left\|v_{h}-\Pi v_{h}\right\|+\left\|V_{\theta} v_{h}-V_{\theta} \Pi v_{h}\right\|+\left\|\nabla_{\hat{s}, t}\left(v_{h}-\Pi v_{h}\right)\right\| \leq C h^{1 / 4-2 \eta}\left\|v_{h}\right\|,
$$

where $\Pi$ is the projection on $u_{\theta}$ defined by:

$$
\Pi \psi(\hat{\tau}, \hat{s}, \hat{t})=\left\langle\psi, u_{\theta}\right\rangle_{\hat{s}, \hat{t}} u_{\theta}(\hat{s}, \hat{t})
$$

In particular, $\Pi: \mathfrak{F}_{N}(h) \rightarrow \Pi\left(\mathfrak{F}_{N}(h)\right)$ is an isomorphism (sometimes called FeshbachGrushin projection).

Proof: As usual we start to prove the inequality when $v_{h}$ is the image of an eigenfunction associated with $\lambda_{p}(h)$, the extension to $v_{h} \in \mathfrak{F}_{N}(h)$ being standard. We want to estimate

$$
\left\|\left(H^{\mathrm{Neu}}(\theta)-\sigma(\theta)\right) v_{h}\right\|
$$

We have:

$$
\left\|\left(H^{\mathrm{Neu}}(\theta)-\sigma(\theta)\right) v_{h}\right\| \leq\left\|\left(H^{\mathrm{Neu}}(\theta)-\lambda_{p}(h)\right) v_{h}\right\|+C h^{1 / 4}\left\|v_{h}\right\| .
$$

With the definition of $v_{h}$ and with Corollary 2.7, we have:

$$
\left\|\left(H^{\mathrm{Neu}}(\theta)-\lambda_{p}(h)\right) v_{h}\right\| \leq h\left\|H_{1} v_{h}\right\|+h^{2}\left\|H_{2} v_{h}\right\|+O\left(h^{\infty}\right)\left\|v_{h}\right\| .
$$

Then, we can write:

$$
\left\|H_{1} v_{h}\right\| \leq C\left\|\hat{t} V_{\theta}\left(D_{\hat{\tau}}-\frac{D_{\hat{s}}}{\sin \theta}\right)^{2} v_{h}\right\|+C\left\|\hat{t}\left(D_{\hat{\tau}}-\frac{D_{\hat{s}}}{\sin \theta}\right)^{2} V_{\theta} v_{h}\right\|+C\left\|\hat{t} V_{\theta}\left(\hat{s}+\frac{\hat{\tau}}{\sin \theta}\right)^{2} v_{h}\right\|
$$

With Lemma 3.2 and the support of $u_{h}$, we infer:

$$
h\left\|H_{1} v_{h}\right\| \leq C h^{1 / 4-2 \eta}\left\|v_{h}\right\| .
$$

In the same way, we get:

$$
h^{2}\left\|H_{2} v_{h}\right\| \leq C h^{1 / 2-4 \eta}\left\|v_{h}\right\|
$$

We deduce:

$$
\left\|\left(H^{\mathrm{Neu}}(\theta)-\sigma(\theta)\right) v_{h}\right\| \leq C h^{1 / 4-2 \eta}\left\|v_{h}\right\|
$$

Let us write

$$
v_{h}=v_{h}^{\perp}+\Pi v_{h}
$$

We have:

$$
\left\|\left(H^{\mathrm{Neu}}(\theta)-\sigma(\theta)\right) v_{h}^{\perp}\right\| \leq C h^{1 / 4-2 \eta}\left\|v_{h}\right\|
$$

The resolvent, valued in the form domain, being bounded, the result follows. 


\section{Harmonic oscillator and last refinements}

In this section, we prove Theorem 1.4. In order to do that, we first prove a localization with respect to $\hat{\tau}$ and then use it to improve the approximation of Proposition 3.9.

\subsection{Control of $v_{h}$ with respect to $\hat{\tau}$}

This subsection is devoted to the reduction to a model operator involving an harmonic oscillator.

Proposition 4.1 There exist $h_{0}>0$ and $C>0$ such that for all $C_{0}>0$ and $h \in\left(0, h_{0}\right)$ :

$$
\begin{aligned}
q_{h}\left(v_{h}\right) \geq & \left(1-C_{0} h\right)\left(\left\|D_{\hat{t}} v_{h}\right\|^{2}+\left\|D_{\hat{s}} v_{h}\right\|^{2}+\left\|\left(V_{\theta}(\hat{s}, \hat{t})+\alpha h \hat{t} H_{\text {harm }}\right) v_{h}\right\|^{2}\right) \\
& -\frac{C}{C_{0}} h\left\langle H_{\text {harm }} v_{h}, v_{h}\right\rangle-C h\left\|v_{h}\right\|^{2},
\end{aligned}
$$

for $v_{h} \in \mathfrak{F}_{N}(h)$.

Proof: Let us consider

$$
q_{h}\left(v_{h}\right)=\left\|D_{\hat{t}} v_{h}\right\|^{2}+\left\|D_{\hat{s}} v_{h}\right\|^{2}+\left\|\left(V_{\theta}(\hat{s}, \hat{t})+\alpha h \hat{t}\left\{H_{\text {harm }}+L\left(\hat{\tau}, D_{\hat{\tau}}, \hat{s}, D_{\hat{s}}\right)\right\}\right) v_{h}\right\|^{2} .
$$

where

$$
L\left(\hat{\tau}, D_{\hat{\tau}}, \hat{s}, D_{\hat{s}}\right)=(\sin \theta)^{-2}\left(-2 \sin \theta D_{\hat{s}} D_{\hat{\tau}}+2 \sin \theta \hat{s} \hat{\tau}+D_{\hat{s}}^{2}+\hat{s}^{2}\right) .
$$

For all $\varepsilon>0$, we have:

$$
\begin{aligned}
q_{h}\left(v_{h}\right) \geq & (1-\varepsilon)\left(\left\|D_{\hat{t}} v_{h}\right\|^{2}+\left\|D_{\hat{s}} v_{h}\right\|^{2}+\left\|\left(V_{\theta}(\hat{s}, \hat{t})+\alpha h \hat{t} H_{\text {harm }}\right) v_{h}\right\|^{2}\right) \\
& -\varepsilon^{-1} \alpha^{2} h^{2}\left\|\hat{t} L\left(\hat{\tau}, D_{\hat{\tau}}, \hat{s}, D_{\hat{s}}\right) v_{h}\right\|^{2}
\end{aligned}
$$

We take $\varepsilon=C_{0} h$. We apply Lemmas $3.3,3.7$ and 3.8 to get:

$$
\left\|\hat{t} L\left(\hat{\tau}, D_{\hat{\tau}}, \hat{s}, D_{\hat{s}}\right) v_{h}\right\|^{2} \leq C\left(\left\|D_{\hat{\tau}} v_{h}\right\|^{2}+\left\|\hat{\tau} v_{h}\right\|^{2}+\left\|v_{h}\right\|^{2}\right) .
$$

A model operator From the last proposition, we are led to study the model operator:

$$
M_{h}=D_{\hat{s}}^{2}+D_{\hat{t}}^{2}+\left(V_{\theta}(\hat{s}, \hat{t})+\alpha h \hat{t} H_{\text {harm }}\right)^{2} .
$$

We can write $M_{h}$ as a direct sum:

$$
M_{h}=\bigoplus_{n \geq 1} M_{h}^{n}
$$


with

$$
M_{h}^{n}=D_{\hat{s}}^{2}+D_{\hat{t}}^{2}+\left(V_{\theta}(\hat{s}, \hat{t})+\alpha h \hat{t} \mu_{n}\right)^{2},
$$

so that, we shall analyze:

$$
H(\theta, \gamma)=D_{\hat{s}}^{2}+D_{\hat{t}}^{2}+\left(V_{\theta}(\hat{s}, \hat{t})+\gamma \hat{t}\right)^{2}
$$

From [5, Proposition 5.1], we deduce the existence of $c>0$ such that, for all $\gamma \geq 0$ :

$$
\sigma(\theta, \gamma) \geq \sigma(\theta)+c \gamma
$$

Taking $C_{0}$ large enough in Proposition 4.1, we deduce the proposition:

Proposition 4.2 There exist $C>0$ and $h_{0}>0$ such that:

$$
\left\langle H_{\text {harm }} v_{h}, v_{h}\right\rangle \leq C\left\|v_{h}\right\|^{2}, \text { for } v_{h} \in \mathfrak{F}_{N}(h)
$$

and:

$$
\lambda_{N}^{\text {resc }}(h) \geq \sigma(\theta)-C h .
$$

\subsection{Refined approximation of $v_{h}$}

The control of $v_{h}$ with respect to $\hat{\tau}$ provided by Proposition 4.2 permits to improve the approximation of $v_{h}$.

Proposition 4.3 There exist $C>0, h_{0}>0$ and $\gamma>0$ such that, if $h \in\left(0, h_{0}\right)$ :

$$
\begin{aligned}
& \left\|V_{\theta} D_{\hat{\tau}} v_{h}-V_{\theta} D_{\hat{\tau}} \Pi v_{h}\right\|+\left\|D_{\hat{\tau}} v_{h}-D_{\hat{\tau}} \Pi v_{h}\right\|+\left\|\nabla_{\hat{s}, \hat{t}}\left(D_{\hat{\tau}} v_{h}-D_{\hat{\tau}} \Pi v_{h}\right)\right\| \leq C h^{\gamma}\left\|v_{h}\right\|, \\
& \left\|V_{\theta} \hat{\tau} v_{h}-V_{\theta} \hat{\tau} \Pi v_{h}\right\|+\left\|\hat{\tau} v_{h}-\hat{\tau} \Pi v_{h}\right\|+\left\|\nabla_{\hat{s}, \hat{t}}\left(\hat{\tau} v_{h}-\hat{\tau} \Pi v_{h}\right)\right\| \leq C h^{\gamma}\left\|v_{h}\right\|,
\end{aligned}
$$

for $v_{h} \in \mathfrak{F}_{N}(h)$.

Proof:

Control with respect to $D_{\hat{\tau}} \quad$ Let us apply $D_{\hat{\tau}}$ to (2.7). We have the existence of $\gamma>0$ such that:

$$
\left\|\left[\mathcal{L}^{\text {Normal }}(h), D_{\hat{\tau}}\right] v_{h}\right\| \leq C h^{\gamma}\left\|v_{h}\right\|
$$

We can write:

$$
\left\|\left(\mathcal{H}^{\mathrm{Neu}}(\theta)-\sigma(\theta)\right) D_{\hat{\tau}} v_{h}\right\| \leq\left\|\left(\mathcal{H}^{\mathrm{Neu}}(\theta)-\lambda_{p}^{\text {resc }}(h)\right) D_{\hat{\tau}} v_{h}\right\|+C h^{1 / 4}\left\|D_{\hat{\tau}} v_{h}\right\| .
$$

Proposition 4.2 provides:

$$
\left\|\left(\mathcal{H}^{\mathrm{Neu}}(\theta)-\sigma(\theta)\right) D_{\hat{\tau}} v_{h}\right\| \leq\left\|\left(\mathcal{H}^{\mathrm{Neu}}(\theta)-\lambda_{p}^{\text {resc }}(h)\right) D_{\hat{\tau}} v_{h}\right\|+C h^{1 / 4}\left\|v_{h}\right\| .
$$


Then, we get:

$$
\left\|h H_{1} D_{\hat{\tau}} v_{h}\right\| \leq C h^{1 / 4-2 \eta}\left\|v_{h}\right\|
$$

and:

$$
\left\|h^{2} H_{2} D_{\hat{\tau}} v_{h}\right\| \leq C h^{1 / 2-4 \eta}\left\|v_{h}\right\|
$$

We deduce:

$$
\left\|\left(\mathcal{H}^{\mathrm{Neu}}(\theta)-\sigma(\theta)\right) D_{\hat{\tau}} v_{h}\right\| \leq C h^{1 / 4-\eta}\left\|v_{h}\right\| .
$$

The conclusion is the same as for the proof of Proposition 3.9.

Control with respect to $\hat{\tau}$ The analysis for $\hat{\tau}$ can be done exactly in the same way.

Conclusion: proof of Theorem 1.4 We recall that:

$$
q_{h}^{\text {Normal }}\left(v_{h}\right)=\left\|D_{\hat{t}} v_{h}\right\|^{2}+\left\|D_{\hat{s}} v_{h}\right\|^{2}+\left\|\left(V_{\theta}(\hat{s}, \hat{t})+\alpha h \hat{t}\left\{H_{\text {harm }}+L\left(\hat{\tau}, D_{\hat{\tau}}, \hat{s}, D_{\hat{s}}\right)\right\}\right) v_{h}\right\|^{2}
$$

so that we get:

$$
\begin{aligned}
& q_{h}^{\text {Normal }}\left(v_{h}\right) \geq\left\langle H(\theta) v_{h}, v_{h}\right\rangle \\
& +\alpha h\left\langle 2 \hat{t} V_{\theta}(\hat{s}, \hat{t}) H_{\text {harm }}+\hat{t} V_{\theta} L\left(\hat{\tau}, D_{\hat{\tau}}, \hat{s}, D_{\hat{s}}\right)+\hat{t} L\left(\hat{\tau}, D_{\hat{\tau}}, \hat{s}, D_{\hat{s}}\right) V_{\theta}(\hat{s}, \hat{t}) v_{h}, v_{h}\right\rangle
\end{aligned}
$$

It remains to approximate $v_{h}$ by $\Pi v_{h}$ modulo a lower order term.

Approximation of the terms involving $H_{\text {harm }}$ Let us examine first:

$$
\left\langle\hat{t} V_{\theta}(\hat{s}, \hat{t}) D_{\hat{\tau}} v_{h}, D_{\hat{\tau}} v_{h}\right\rangle=\left\langle\hat{t} D_{\hat{\tau}} v_{h}, V_{\theta} D_{\hat{\tau}} v_{h}\right\rangle \text {. }
$$

We have:

$$
\left\|\hat{t} D_{\hat{\tau}} v_{h}\right\| \leq C\left\|v_{h}\right\|
$$

and thus, with Proposition 4.3:

$$
\left\langle\hat{t} D_{\hat{\tau}} v_{h}, V_{\theta} D_{\hat{\tau}} v_{h}\right\rangle=\left\langle\hat{t} D_{\hat{\tau}} v_{h}, V_{\theta} \Pi D_{\hat{\tau}} v_{h}\right\rangle+o(1)\left\|v_{h}\right\|^{2} .
$$

We can write:

$$
\left\|\hat{t} V_{\theta} \Pi D_{\hat{\tau}} v_{h}\right\| \leq C\left\|v_{h}\right\|
$$

and it follows:

$$
\left\langle\hat{t} D_{\hat{\tau}} v_{h}, V_{\theta} \Pi D_{\hat{\tau}} v_{h}\right\rangle=\left\langle\Pi D_{\hat{\tau}} v_{h}, \hat{t} V_{\theta} \Pi D_{\hat{\tau}} v_{h}\right\rangle+o(1)\left\|v_{h}\right\|^{2} .
$$

We deduce that:

$$
\left\langle\hat{t} V_{\theta}(\hat{s}, \hat{t}) D_{\hat{\tau}} v_{h}, D_{\hat{\tau}} v_{h}\right\rangle=\left\langle\hat{t} V_{\theta}(\hat{s}, \hat{t}) D_{\hat{\tau}} \Pi v_{h} D_{\hat{\tau}} \Pi v_{h}\right\rangle+o(1)\left\|v_{h}\right\|^{2} .
$$

Let us consider:

$$
\left\langle\hat{t} V_{\theta}(\hat{s}, \hat{t}) \hat{\tau} v_{h}, \hat{\tau} v_{h}\right\rangle=\left\langle\hat{t} \hat{\tau} v_{h}, V_{\theta} \hat{\tau} v_{h}\right\rangle
$$

In order to treat this term, we use:

$$
\left\|\hat{t} \hat{\tau} v_{h}\right\| \leq C\left\|v_{h}\right\| .
$$


Approximations of the other terms Another term we should approximate is:

$$
\left\langle\hat{t} V_{\theta}(\hat{s}, \hat{t}) D_{\hat{s}} v_{h}, D_{\hat{\tau}} v_{h}\right\rangle=\left\langle\hat{t} D_{\hat{s}} v_{h}, V_{\theta}(\hat{s}, \hat{t}) D_{\hat{\tau}} v_{h}\right\rangle
$$

We can notice that:

$$
\left\|\hat{t} D_{\hat{s}} v_{h}\right\| \leq C\left\|v_{h}\right\|
$$

so that:

$$
\left\langle\hat{t} D_{\hat{s}} v_{h}, V_{\theta}(\hat{s}, \hat{t}) D_{\hat{\tau}} v_{h}\right\rangle=\left\langle\hat{t} D_{\hat{s}} v_{h}, V_{\theta}(\hat{s}, \hat{t}) \Pi D_{\hat{\tau}} v_{h}\right\rangle+o(1)\left\|v_{h}\right\|^{2}
$$

We write:

$$
\left\langle\hat{t} D_{\hat{s}} v_{h}, V_{\theta}(\hat{s}, \hat{t}) \Pi D_{\hat{\tau}} v_{h}\right\rangle=\left\langle D_{\hat{s}} v_{h}, \hat{t} V_{\theta}(\hat{s}, \hat{t}) \Pi D_{\hat{\tau}} v_{h}\right\rangle
$$

and use:

$$
\left\|\hat{t} V_{\theta}(\hat{s}, \hat{t}) \Pi D_{\hat{\tau}} v_{h}\right\| \leq C\left\|v_{h}\right\|
$$

to find:

$$
\left\langle D_{\hat{s}} v_{h}, \hat{t} V_{\theta}(\hat{s}, \hat{t}) \Pi D_{\hat{\tau}} v_{h}\right\rangle=\left\langle D_{\hat{s}} \Pi v_{h}, \hat{t} V_{\theta}(\hat{s}, \hat{t}) \Pi D_{\hat{\tau}} v_{h}\right\rangle+o(1)\left\|v_{h}\right\|^{2}
$$

In the same way, we find:

$$
\begin{aligned}
& \left\langle\hat{t} \hat{s} v_{h}, V_{\theta} \hat{\tau} v_{h}\right\rangle=\left\langle\hat{t} \hat{s} \Pi v_{h}, V_{\theta} \hat{\tau} \Pi v_{h}\right\rangle+o(1)\left\|v_{h}\right\|^{2}, \\
& \left\langle\hat{t} \hat{s}^{2} v_{h}, V_{\theta} v_{h}\right\rangle=\left\langle\hat{t} \hat{s}^{2} \Pi v_{h}, V_{\theta} \Pi v_{h}\right\rangle+o(1)\left\|v_{h}\right\|^{2}, \\
& \left\langle\hat{t} D_{\hat{s}}^{2} v_{h}, V_{\theta} v_{h}\right\rangle=\left\langle\hat{t} D_{\hat{s}}^{2} \Pi v_{h}, V_{\theta} \Pi v_{h}\right\rangle+o(1)\left\|v_{h}\right\|^{2} .
\end{aligned}
$$

We infer:

$$
\begin{aligned}
q_{h}^{\text {Normal }}\left(v_{h}\right) & \geq \sigma(\theta)\left\|v_{h}\right\|^{2} \\
& +\alpha h\left\langle 2 \hat{t} V_{\theta}(\hat{s}, \hat{t}) H_{\text {harm }}+\hat{t} V_{\theta} L\left(\hat{\tau}, D_{\hat{\tau}}, \hat{s}, D_{\hat{s}}\right)+\hat{t} L\left(\hat{\tau}, D_{\hat{\tau}}, \hat{s}, D_{\hat{s}}\right) V_{\theta}(\hat{s}, \hat{t}) \Pi v_{h}, \Pi v_{h}\right\rangle \\
& +o(h)\left\|v_{h}\right\|^{2} .
\end{aligned}
$$

This implies:

$$
q_{h}^{\text {Normal }}\left(v_{h}\right) \geq \sigma(\theta)\left\|v_{h}\right\|^{2}+\alpha h\left\langle\mathfrak{S}_{\theta}\left(D_{\hat{\tau}}, \hat{\tau}\right) \phi_{h}, \phi_{h}\right\rangle_{\hat{\tau}}+o(h)\left\|v_{h}\right\|^{2},
$$

where $\phi_{h}=\left\langle v_{h}, u_{\theta}\right\rangle_{\hat{s}, \hat{t}}$ and $v_{h} \in \mathfrak{F}_{N}(h)$. We infer:

$$
h^{-1}\left(\lambda_{N}(h)-\sigma(\theta)+o(h)\right)\left\|v_{h}\right\|^{2} \geq \alpha\left\langle\boldsymbol{S}_{\theta}\left(D_{\hat{\tau}}, \hat{\tau}\right) \phi_{h}, \phi_{h}\right\rangle_{\hat{\tau}}
$$

With Proposition 3.9 and the min-max principle, we deduce that:

$$
\lambda_{N}(h) \geq \sigma(\theta)+\alpha h \nu_{N}\left(\mathfrak{S}_{\theta}\left(D_{\hat{\tau}}, \hat{\tau}\right)\right)+o(h) .
$$

This provides the spectral gap between the lowest eigenvalues. Jointly with Theorem 1.3, we deduce Theorem 1.4. 
Acknowledgments The author would like to thank Soeren Fournais for his invitation at the University of Aarhus (Winter 2010) where the first ideas of this paper were discussed. The author is also grateful to Mikael Persson for many useful discussions.

\section{References}

[1] S. Agmon. Lectures on exponential decay of solutions of second-order elliptic equations: bounds on eigenfunctions of $N$-body Schrödinger operators, volume 29 of Mathematical Notes. Princeton University Press, Princeton, NJ 1982.

[2] S. Agmon. Bounds on exponential decay of eigenfunctions of Schrödinger operators. In Schrödinger operators (Como, 1984), volume 1159 of Lecture Notes in Math., pages 1-38. Springer, Berlin 1985.

[3] P. Bauman, D. Phillips, Q. TANG. Stable nucleation for the Ginzburg-Landau system with an applied magnetic field. Arch. Rational Mech. Anal. 142(1) (1998) $1-43$.

[4] A. Bernoff, P. Sternberg. Onset of superconductivity in decreasing fields for general domains. J. Math. Phys. 39(3) (1998) 1272-1284.

[5] V. Bonnaillie, M. Dauge, N. Popoff, N. Raymond. Discrete spectrum of a model schrödinger operator on the half-plane with neumann conditions. Z. Ange. Math. Phys. (2011).

[6] H. L. CyCOn, R. G. Froese, W. Kirsch, B. Simon. Schrödinger operators with application to quantum mechanics and global geometry. Texts and Monographs in Physics. Springer-Verlag, Berlin, study edition 1987.

[7] M. Del Pino, P. L. Felmer, P. Sternberg. Boundary concentration for eigenvalue problems related to the onset of superconductivity. Comm. Math. Phys. 210(2) (2000) 413-446.

[8] N. Dombrowski, N. RAYmond. Semiclassical analysis with vanishing magnetic fields. Preprint (2012).

[9] S. Fournais, B. HelfFER. Accurate eigenvalue asymptotics for the magnetic Neumann Laplacian. Ann. Inst. Fourier (Grenoble) 56(1) (2006) 1-67.

[10] S. Fournais, B. HelfFER. Spectral methods in surface superconductivity. Progress in Nonlinear Differential Equations and their Applications, 77. Birkhäuser Boston Inc., Boston, MA 2010.

[11] V. V. GRUŠIN. Hypoelliptic differential equations and pseudodifferential operators with operator-valued symbols. Mat. Sb. (N.S.) 88(130) (1972) 504-521. 
[12] B. HELFFER. Semi-classical analysis for the Schrödinger operator and applications, volume 1336 of Lecture Notes in Mathematics. Springer-Verlag, Berlin 1988.

[13] B. HelfFer, Y. A. Kordyukov. Spectral gaps for periodic Schrödinger operators with hypersurface magnetic wells: analysis near the bottom. J. Funct. Anal. 257(10) (2009) 3043-3081.

[14] B. Helffer, A. Mohamed. Semiclassical analysis for the ground state energy of a Schrödinger operator with magnetic wells. J. Funct. Anal. 138(1) (1996) 40-81.

[15] B. Helffer, A. Morame. Magnetic bottles in connection with superconductivity. J. Funct. Anal. 185(2) (2001) 604-680.

[16] B. Helffer, A. Morame. Magnetic bottles for the Neumann problem: the case of dimension 3. Proc. Indian Acad. Sci. Math. Sci. 112(1) (2002) 71-84. Spectral and inverse spectral theory (Goa, 2000).

[17] B. Helffer, A. Morame. Magnetic bottles for the Neumann problem: curvature effects in the case of dimension 3 (general case). Ann. Sci. École Norm. Sup. (4) 37(1) (2004) 105-170.

[18] K. Lu, X.-B. PAN. Eigenvalue problems of Ginzburg-Landau operator in bounded domains. J. Math. Phys. 40(6) (1999) 2647-2670.

[19] K. LU, X.-B. PAN. Surface nucleation of superconductivity in 3-dimensions. J. Differential Equations 168(2) (2000) 386-452. Special issue in celebration of Jack K. Hale's 70th birthday, Part 2 (Atlanta, GA/Lisbon, 1998).

[20] R. Montgomery. Hearing the zero locus of a magnetic field. Comm. Math. Phys. 168(3) (1995) 651-675.

[21] X.-B. PAN, K.-H. KWEK. Schrödinger operators with non-degenerately vanishing magnetic fields in bounded domains. Trans. Amer. Math. Soc. 354(10) (2002) 42014227 (electronic).

[22] A. Persson. Bounds for the discrete part of the spectrum of a semi-bounded Schrödinger operator. Math. Scand. 8 (1960) 143-153.

[23] N. RAYMOND. Sharp asymptotics for the Neumann Laplacian with variable magnetic field: case of dimension 2. Ann. Henri Poincaré 10(1) (2009) 95-122.

[24] N. RAYMOND. On the semiclassical 3D Neumann Laplacian with variable magnetic field. Asymptot. Anal. 68(1-2) (2010) 1-40. 